\title{
Feature Extraction of Single Face Image Based Active Appearance Model
}

\author{
Zhiqiang Cao \\ School of Science, Wuhan University of Technology Wuhan, China
}

\begin{abstract}
Face as the most direct and important carrier of human's daily emotional expression and information exchange, with its peculiar to different from other organs and parts of the universality and ease of use as mathematical modeling of the object of study, and many research algorithm has been applied to the photographing, face recognizing and face rebuilding, etc. Currently, there are face recognizing and face rebuilding two research direction around face, this paper studies the AAM algorithm based on front face feature points extraction, and using MATLAB to achieve programming. Results show that this method can quickly locate face to extract the complete face shape.
\end{abstract}

Keywords-single image; active appearance model; face recognition; facial feature extraction

\section{INTRODUCTION}

People's face is different from individual memory different characters is the most important content, and is the carrier of expressing joys and sorrows [1]. In real life, there are different kinds of method to confirm people's identity, such as voice, iris and fingerprints, etc. Compared to other methods face image is more convenient to achieve. In recent decades, to the face image for processing object of study includes many fields, such as face detection, face tracking, facial feature location, face recognition, facial animation and facial expression recognition, etc. With the hard work of many researchers, many areas has made a series of research achievements, and obtained a certain degree of practical application.

\section{RESEARCH BACKGROUND}

Nowadays there are a huge number of method to build the 3 $\mathrm{d}$ face model, according to the $3 \mathrm{~d}$ face information sources, these methods can be roughly divided into two groups. One kind is directly obtained, through $3 \mathrm{~d}$ digital scanning equipment of face scanning to obtain three dimensions face information and facial texture color information. Another kind is indirect gain, first returning face to $2 \mathrm{~d}$ images by cameras, then through the computer processing to get the threedimensional information and texture information, etc [2, 3, 4, 5].

Geting $3 \mathrm{~d}$ face information through two-dimensional pictures is relatively easy, using two-dimensional face image and some algorithms tools can set up $3 \mathrm{~d}$ face model [3]. According to the number of images used in the process of $3 \mathrm{~d}$ face reconstruction, the indirect method can be divided into single image $3 \mathrm{~d}$ reconstruction and multiple view image $3 \mathrm{~d}$ reconstruction. Single image $3 \mathrm{~d}$ reconstruction refers to the method of recovering $3 \mathrm{~d}$ information from single $2 \mathrm{~d}$ image.
Because the required conditions of single image threedimensional reconstruction is low, it has a broad application prospect.

\section{PRINCIPLE AND ALGORITHM}

\section{A. Research Status}

Facial feature points positioning is the indispensable step to realize the automation in the process of $3 \mathrm{~d}$ face modeling [6]. At present commonly used face feature points location models are AdaBoost model, active shape model (ASM) and active appearance model (AAM), etc.

AdaBoost model analyzes the target face image first, determining whether there is a face region, the characteristics using in analysis process is the Harr characteristic. Then using the characteristic to create a large number of suitable weak classifiers. These classifiers separate ability are general, but by proper methods they can stack to a stronger classifier. In the end, the stronger classifier cascade as classifier algorithm in practical application [7-10]. In practice, however, AdaBoost model accuracy is not high [7].

ASM and AAM model all study a change of the shape by estimating the statistical law of training data set. ASM model position feature points in the image relying on global shape model and local appearance model of each feature point. For AAM model, it establishs global shape model and texture model, through the shape changes in the image sampling, make the sampling image texture and texture model to match $[6,7,11-$ 14]. ASM model is affected deeply by the initial value, if the initial value is near the global optimal solution, ASM model can be fast convergent. On the contrary it is likely to fall into local optimal solution. AAM model has high precision only if under the condition of the small disturbance, thus it limits the scope of application of the model.

In conclusion, this article mainly uses the active appearance model for face feature extraction.

\section{B. AAM Model Algorithm}

Active appearance model is put forward by Cootes and others [15] at the automatic human face and gesture recognition international conference, it is a combinedation model of shape changes and texture changes information model. AAM model can be thought of as the combination of shape information model and texture information model. Shape information refers to the pure geometry graphics of the image, without the scale, translation and rotation factors. Texture information is the color 
or brightness values of pixel in interested area. The first step to establish AAM model is to treat the image which have calibrated feature points' location as the training sample data, then analysising and arranging these points set, and establishing a statistical shape model. Change training image samples to match their average shape, then get a texture image which is nothing on the shape. Finally, adjust these two models to get the AAM model. In typical applications of AAM algorithm, the first step is often fitting the AAM model to the target image to be detected, which means to calculate the parameters of adjusting the model, to make model can exactly match as far as possible to the target area. The fitting of AAM model and the image is a nonlinear optimization problem, usual method for calculating this kind of problem is to iterative update parameter values [16].

\section{1) Shape Information Model}

The shape information of AAM model by can be seen as geometric Figure s made up of $\mathrm{v}$ points, and its mathematical expression is: $s=\left(x_{1}, y_{1}, \mathrm{~L}, x_{v}, y_{v}\right)$, each sample image contains the marked shape area s. For the set of all training images shape s we do principal component analysis, $S_{0}$ is the mean of these sample shape, and $n$ feature vectors of the first $n$ characteristics calculated through principal components can be composed of matrix $S_{i}$, then any sample shape s can be obtained by the following formula: [7]

$$
S=s_{0}+\sum_{i=1}^{n} p_{i} s_{i}
$$

$P_{i}$ is the shape parameter. Before PCA, it needs to align the sample image shape with the average shape, and normalize training samples. It can remove transformation produced by choosing global shape deformation to normalize.

\section{2) Texture Information Model}

The texture information refers to the color or brightness information of image in average shape $S_{0}$. The corresponding image in pixel $\mathbf{x}=(x, y)^{T} \in s_{0}$ is $A(\mathbf{x})$. By the same method as processing shape information, we do principal component analysis with the set of all training images, then get the mean texture $A_{0}$ and matrix $A_{i}$ consisted of the set of $m$ principal component characteristic vectors. In the same way, any texture information $A(\mathbf{x})$ in the sample set can be expressed by the following formula: [7]

$$
A(X)=A_{0}(X)+\sum_{i=1}^{m} q_{i} A_{i}(X), \forall X \in S_{0}
$$

\section{$q_{i}$ is the texture parameters.}

\section{3) Modeling}

Known the shape parameter $p_{i}=\left(p_{1}, \mathrm{~L}, p_{n}\right)^{T}$ and the texture parameters $q_{i}=\left(q_{1}, \mathrm{~L}, q_{m}\right)^{T}$, according to the formula (1) and (2) we can determine the shape of the AAM model and the corresponding texture model. The establishment of AAM model is to plug the mean shape $S_{0}$ and the texture information $A_{0}$ into the model shape $\mathrm{s}$, and obtain the parameters $\mathrm{p}$ and $\mathrm{q}$. The process of plugging can be defined by the following equation: $M(W(X ; P))=A(X) \quad \mathrm{M}$ is a appropriate size and shape two-dimensional image which contains model instance. $\mathrm{W}(\mathrm{X} ; \mathrm{P})$ is a function of deformation texture. Known a pixel $\mathrm{x}$ in shape $S_{0}$, the target value of the deformation is $\mathrm{W}(\mathrm{x} ; \mathrm{p})$. For AAM model $\mathrm{M}$, the texture value of the pixel $\mathrm{W}(\mathrm{x} ; \mathrm{p})$ in its shape model s is $A(\mathbf{x})$.

\section{4) Model Fitting Image}

Assume that the input image is $I(X)$, now we need to fitting it to the AAM model known optimal shape parameters $p$ and texture parameter $\mathrm{q}$, this means that the input image I and AAM model instance $M(W(X ; P))=A(X)$ must be similar. In order to define the fitting process, first we need to define a standard of optimization, obviously, it means to minimize the error between the input image $I(X)$ and instance $M(W(X ; P))=A(X)$. Here the error, it can be calculated in two coordinate space, image coordinate and AAM model coordinate. Considering the efficiency of the fitting method, here we choose the coordinates of AAM model, the corresponding coordinate in pixel $\mathbf{X}$ from the average shape $S_{0}$ of AAM model is $\mathrm{W}(\mathrm{x} ; \mathrm{p})$, the texture of pixel $\mathbf{x}$ in AAM model is

$$
A(\mathbf{x})=A_{0}(\mathbf{x})+\sum_{i=1}^{m} q_{i} A_{i}(\mathbf{x})
$$
image texture information is $I(W(\mathbf{x} ; p))$, here we need to minimize the square variance of the two kinds of texture information: [7]

$$
\sum_{\mathbf{x} \in s_{0}}\left[A_{0}(\mathbf{x})+\sum_{i=1}^{m} q_{i} A_{i}(\mathbf{x})-I(W(\mathbf{x} ; p))\right]^{2}
$$

Here we define an error image $E(\mathbf{x})$,

$$
E(\mathbf{x})=A_{0}(\mathbf{x})+\sum_{i=1}^{m} q_{i} A_{i}(\mathbf{x})-I(W(\mathbf{x} ; p))
$$

[7]. For each 
pixel $\mathbf{X}$ in the mean shape $S_{0}$, we use piecewise affine transformation $\mathrm{W}$ to calculate the corresponding pixel point $\mathrm{W}(\mathrm{x} ; \mathrm{p})$ in the input image. The input image I using bilinear interpolation of pixel point $W(\mathbf{x} ; p)$ calculation sample, from the point of AAM texture model values we minus the interpolation to calculate the results stored in $\mathrm{E}$.

\section{TEXT AND RESUltS}

First of all, collect $n$ positive face photos as sample, use ginput function in MATLAB to marking key feature points in each image (see Figure 1).

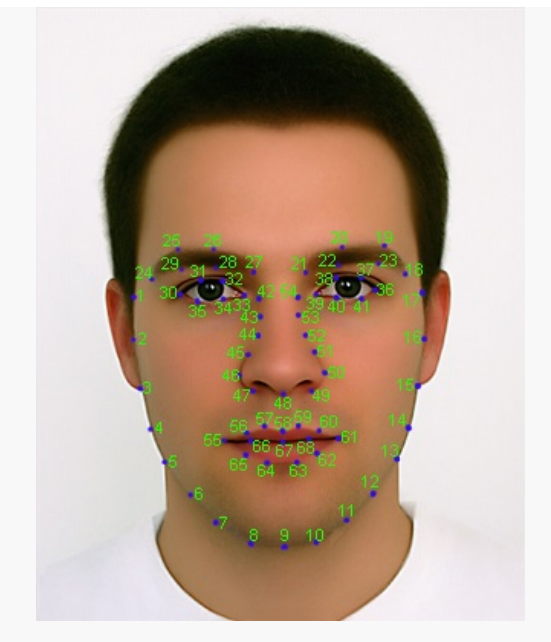

FIGURE I. KEY FEATURE POINTS’ MARKING

Afterwards, use v key feature points in each Figure to form a shape vector, so result $n$ shape vectors.Then use Procrustes method to normalize the shape vectors, thereby eliminating the disturbance of external factors in the image, such as: face angle, face distance and posture etc. Finally make PCA handling with the aligned vectors to get the average shape vector. In the same way, the average texture of the image can also be obtained (see Figure 2).

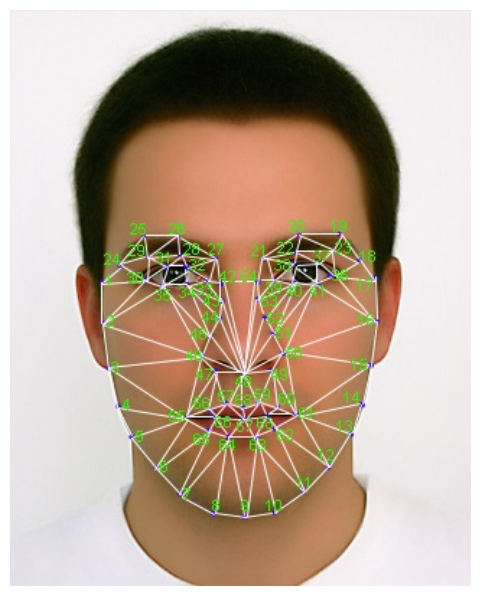

FIGURE II. THE AVERAGE TEXTURE
We use MATLAB to programming AAM algorithm to realize the automation of single face image feature extraction. Here, we cab only extract eyes and lips feature points with the mouse in turns at the selected image manually, the program will automatically use the average characteristics model to make the affine transformation by these three points, then we get the corresponding face shape model and texture model (see Figure 3).

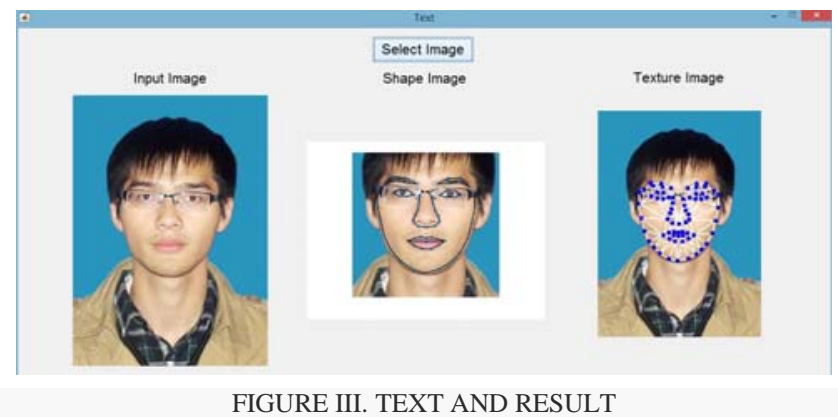

\section{CONCLUSION}

This paper has studied the active appearance model, and used MATLAB programming to realize the automation of single face image feature extraction. The program operation is simple, all we need is to manually locate center of eyes and lips on the input image, then we can complete the face detection and feature point extraction fast. But in terms of algorithm, it remains to be improved. The AAM used in the text is based on the PCA, but PCA only considers the second order statistics of the data, which contains no phase information, and the ability to describe the local characteristics of image is weak. When the model can synthetic most of images correctly, search process will stop. In order to further improve the accuracy, we can further research AAM algorithm based on independent component analysis ICA.

\section{ACKNOWLEDGEMENTS}

This paper is supported by the Fundamental Research Funds for the Central Universities (WUT: 2016-IA-004) and Students Innovation and Entrepreneurship Training Program (No. 20151049714001).

\section{REFERENCES}

[1] ZHANG Cui-ping,SU Guang-da.Human Face Recognition: A Survey[J].Journal of Image and Graphics,2000,5(11):885-894.

[2] Yu Zhao.3D Reconstruction Based on Single Images[D]:Nanjing University of Posts,2013.

[3] Dianbo Li.RESEARCH ON 3D FACE VISUALIZATION BASED ON SINGLE IMAGE[D]: University of Electronic Science and Technology of China,2013.

[4] Li Xueyan,Ma Qian,Li Daming.Research of 3D Modeling Based on a Single 2D Image[J].Software Guide, 2014,(09):160-162.

[5] XIONG Ping,LU Ye.Modified 3D facial reconstruction algorithm based on a single photo[J].Journal of Computer Applications, 2013,(08):2359$2361+2369$.

[6] Ying ZHENG.Image-based 3D Face Modeling[D]:University of Science and Technology of China,2009.

[7] LU Ye. Research and Application on 3D Facial Reconstruction Methods[D]: Central South University, 2013. 
[8] Zhang J,Li J,Jiang S,Wei Z,Li J,Xu S,Wang S.The research of face detection based on double skin model and AdaBoostalgorithm:proceedings of the Conference Anthology,IEEE,2013[C].IEEE.

[9] Phuong HM,Dung L,de Souza-Daw T,Dzung NT,Hoang TM.Extraction of human facial features based on Haar feature with AdaBoostand image recognition techniques:proceedings of the Communications and Electronics(ICCE),2012 Fourth International Conference on,2012[C]. IEEE.

[10] Kim M,Han S.Real-time face detection on reconfigurable device:proceedings of the ICT Convergence(ICTC),2013 International Conference on,2013[C].IEEE.

[11] Hsu W-H,Hsieh C-T,Xu Weixin,Xie Jingtang,Hsu W-H.A Simple Algorithm for 3D Face ReconstructionModel[J].2014.

[12] Behaine CA,Scharcanski J.Enhancing the performance of active shape models in face recognition applications[J].Instrumentation and Measurement,IEEE Transactions on,2012,61(8):2330-2333.

[13] Velayutham M,Shivakumar B.An Exploration on Data Mining for Face Detection based on Real-time Face Tracking[J].Journal of Computer Technology \& Applications,2015,5(3):46-51.

[14] Khan MA,Xydeas C,Ahmed H.On the application ofAAM-based systems in face recognition:proceedings of the Signal Processing Conference (EUSIPCO),2013 Proceedings of the 22nd European,2014[C].IEEE.

[15] Edwards G.J.,Taylor C.J.,Cootes T.F..Interpreting face images using activeappearance models[C].Proceedings of International Conference on AutomaticFace and Gesture Recognition,1998:300-305.

[16] Cootes T.F.,Edwards G.J.,Taylor C.J..Active appearancemodels[J]IEEETransactions on Pattern Analysis and Machine Intelligence, 2001,23(6):681-685. 